\title{
Rattle-Type Silica Colloidal Particles Prepared by a Surface- Protected Etching Process
}

\author{
Qiao Zhang, Jianping Ge, James Goebl, Yongxing Hu, Zhenda Lu, and Yadong Yin（ $\varangle)$ \\ Department of Chemistry, University of California, Riverside, California 92521, USA \\ Received: 10 May 2009 / Accepted: 19 May 2009 \\ CTsinghua University Press and Springer-Verlag 2009. This article is published with open access at Springerlink.com
}

\begin{abstract}
This paper explores the capability of the "surface-protected etching" process for the creation of rattle-type $\mathrm{SiO}_{2} @$ void@SiO $\mathrm{S}_{2}$ colloidal structures featuring a mesoporous silica shell and a mesoporous movable silica core. The surface-protected etching process involves stabilization of the particle surface using a polymer ligand, and then selective etching of the interior to form hollow structures. In this paper, this strategy has been extended to the formation of rattle-like structures by etching $\mathrm{SiO}_{2} @ \mathrm{SiO}_{2}$ core-shell particles which are synthesized by a two-step sol-gel process. The key is to introduce a protecting polymer of polyvinylpyrrolidone (PVP) to the surface of both core and shell in order to tailor their relative stability against chemical etching. Upon reacting with $\mathrm{NaOH}$, the outer layer silica becomes a hollow shell as only the surface layer is protected by PVP and the interior is removed, while the core remains its original size thanks to the protection of PVP on its surface. This process can be carried out at room temperature without the need of additional templates or complicated heterogeneous coating procedures. The etching process also results in the rattle-type colloids having mesoscale pores with two distinct average sizes. In our demonstration of a model drug delivery process, such mesoporous structures show an interesting two-step elution profile which is believed to be related to the unique porous rattle structures.
\end{abstract}

\section{KEYWORDS}

Silica, mesoporous, core-shell structure, surface-protected etching, drug delivery

\section{Introduction}

Recent progress in nanostructure synthesis has made it possible to create inorganic colloidal particles with complex morphologies, compositions, and internal structures. Multiple functionalities can be integrated into individual particles through various chemical processes, making them attractive materials for a wide range of applications in areas such as coatings, optics and electronics, catalysis, separations, and diagnostics. A typical example of such nanostructures includes so-called core-shell particles which are often produced by coating or encapsulating a core particle within a shell of another material [1-4]. Depending on the properties of the core and shell, magnetic, optical, or catalytic functions may be readily imparted to the dispersed colloidal matter. Encapsulation with shells of different compositions may also protect the active

Address correspondence to yadong.yin@ucr.edu 
core material from extraneous chemical and physical changes.

Rattle-type or "yolk-shell" structures, a class of special core-shell structures with a distinctive core@void@shell configuration, have shown many important potential applications that are not accessible to conventional core-shell particles $[5,6]$. For example, a metallic nanoparticle encapsulated in a hollow oxide shell can function as a nanoscale reactor for catalytic reactions [7]. The catalyst cores remain as discrete particles with high specific surface area while the shells, if made mesoporous, allow transport of reactants and products, and at the same time prevent the aggregation of the catalyst particles. Rattle-type particles can also find use as unique drug delivery vehicles, in which the hollow shells provide space for controlled loading of bioactive molecules and the core particle may sense the external stimuli and trigger the release. Typically, the core and shell in a rattle-type particle are made of different materials, e.g., metal core with silica or other oxide shell, or oxide core with polymer or carbon shell [8]. Templating strategies have been widely used for synthesizing rattle-type nanostructures: a sandwich structure (core/template/shell) is fabricated first, and then the sacrificial template layer is selectively removed to form the void. Multiple coating processes are typically involved in the templating methods, making it difficult to control the reproducibility because heterogeneous nucleation and growth are not always preferred. The complicated synthetic procedures have prevented scale-up of many of these methods for actual industrial applications. There are also some reports of the preparation of rattle-type structures from simple core-shell structures. We have previously demonstrated the direct transformation of a solid metal coating into metal oxide or sulfide hollow shells through a chemical process analogous to the classic Kirkendall effect [9]. Voids can also form from a core-shell particle by selective etching of the core particle to decrease its size [10].

Recently, we have developed a "surface-protected etching" process for the direct conversion of solid oxide spheres into hollow structures without using additional templates [7]. Our initial demonstration was focused on silica because this material is important in various fields such as biology, catalysis, health science, and many industrial applications [11 -14]. It has also been widely used in the fabrication of rattle-type core-shell structures, either as a removable template or outer shell [15]. In our system, the hollowing process involves the initial binding of the silica surface with polymer ligands (e.g., polyvinylpyrrolidone, PVP) and the subsequent dissolution of the core with an etching agent (e.g., $\mathrm{NaOH})$. The partial etching of the surface layer produces mesopores in the shell. We demonstrated that by rationally choosing the reaction components, it is possible to generalize this process into a strategy for the production of porous hollow structures of many other materials such as $\mathrm{TiO}_{2}$ [16]. Unlike the traditional soft template-guided synthesis where mesoporous structures are difficult to achieve in monodisperse colloidal samples [17], this etching process is a convenient and effective means to form mesoporous shells, especially for those applications where pore ordering is inessential.

In this paper, we further explore the capability of the "surface-protected etching" strategy and demonstrate a simple process for the synthesis of porous-SiO $@$ @oid@porous-SiO 2 (p-SiO $\mathrm{S}_{2} @$ void@p-SiO${ }_{2}$ ) rattle-type colloidal structures featuring a mesoporous silica shell and a movable and mesoporous silica core. In contrast to the conventional procedures, this process can be carried out at room temperature without the need of additional templates and complicated coating procedures. The key is to firstly grow core-shell silica colloids in two steps so that the protecting polymer can be sandwiched between the two silica layers, and then use surface-protected etching to produce a gap between the core and shell (Fig. 1). This process can be easily reproduced and scaled up because no heterogeneous coating steps are involved. During the hollowing process, the etchant also partially removes materials from the remaining silica. As a result, the as-prepared rattle-type colloids contain mesoscale pores with two distinct average sizes. In our demonstration of a model drug delivery process, such mesoporous structures show an interesting twostep elution profile which is believed to relate to the unique porous rattle-type structures. 


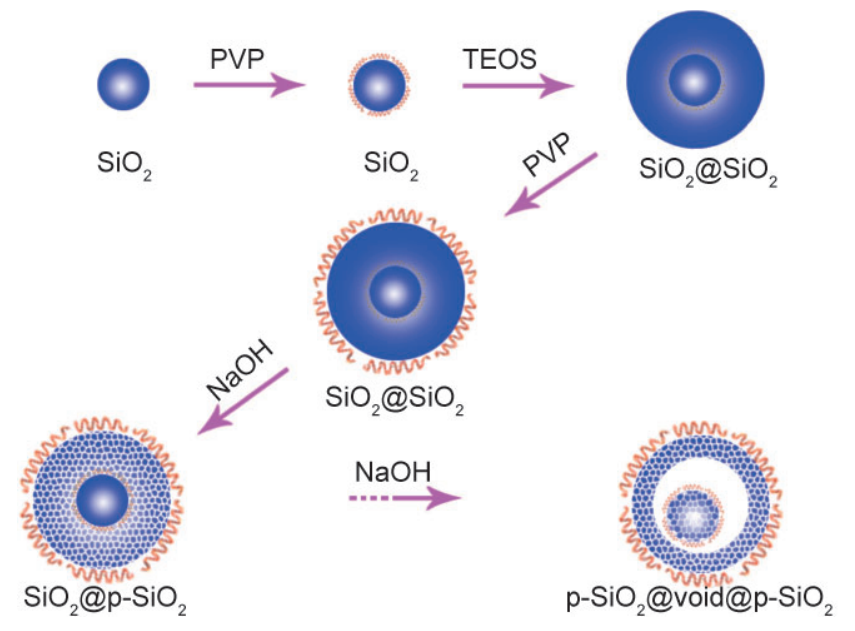

Figure 1 Schematic illustration of the synthesis of $\mathrm{p}-\mathrm{SiO}_{2} @ \mathrm{void@}$ $\mathrm{p}-\mathrm{SiO}_{2}$ colloidal particles through the surface-protected etching process

\section{Experimental}

\subsection{Chemicals}

Ethanol (denatured), ammonium hydroxide aqueous solution $(28 \%)$, and sodium hydroxide were purchased from Fisher Scientific. Tetraethyl orthosilicate (TEOS, 98\%), PVP $\left(M_{\mathrm{w}} \sim 55,000\right)$, and Rhodamine 6G (R6G, 99\%) were obtained from Sigma-Aldrich. PVP K15 $\left(M_{\mathrm{w}} \sim 10,000\right)$ was purchased from Fluka.

\subsection{Synthesis of silica seeds}

Monodisperse $\mathrm{SiO}_{2}$ particles were prepared by using a modified Stöber method [18]. Ammonium hydroxide solution $(2.0 \mathrm{~mL})$, deionized (DI) water $(8.0 \mathrm{~mL})$, and ethanol $(48.0 \mathrm{~mL})$ were mixed in a $250-\mathrm{mL}$ threeneck flask with magnetic stirring. A $50.0 \mathrm{~mL}$ ethanol solution containing $5.0 \mathrm{~mL}$ TEOS was quickly injected into the flask to form a clear solution. The system became turbid in $\sim 1 \mathrm{~h}$, and was then kept at room temperature under continuous magnetic stirring for $24 \mathrm{~h}$. The final product was centrifuged and washed with water and re-dispersed in $30 \mathrm{~mL}$ water. The silica particles have a typical diameter of $\sim 200 \mathrm{~nm}$.

\subsection{PVP pretreatment of silica seeds}

The silica colloidal solution (30 mL) was mixed with PVP $\left(2 \mathrm{~g}, M_{\mathrm{w}} \sim 55,000\right)$ at room temperature under magnetic stirring. After refluxing for $3 \mathrm{~h}$, the solution was then cooled down to room temperature. Finally, the PVP treated silica seeds were collected by centrifugation and re-dispersed in $30 \mathrm{~mL} \mathrm{DI}$ water.

\subsection{Synthesis of $\mathrm{p}-\mathrm{SiO}_{2} @$ void@ $\mathrm{p}-\mathrm{SiO}_{2}$ colloids}

The silica seed solution $(0.5 \mathrm{~mL}$ out of a total of 30 $\mathrm{mL}$ ) was dispersed in $100 \mathrm{~mL}$ ethanol with magnetic stirring, followed by sequential addition of $12 \mathrm{~mL}$ $\mathrm{H}_{2} \mathrm{O}, 5 \mathrm{~mL}$ ammonium hydroxide solution, and $1 \mathrm{~mL}$ TEOS. The mixture was kept at room temperature for $3 \mathrm{~h}$. The product was collected by centrifugation, dispersed in $20 \mathrm{~mL}$ water, mixed with $1 \mathrm{~g}$ PVP K15, and then refluxed for $3 \mathrm{~h}$. Upon cooling the mixture down to room temperature, $\mathrm{NaOH}$ aqueous solution $(5 \mathrm{~mL}, 0.20 \mathrm{~g} / \mathrm{mL}$ ) was injected to the system to initiate etching. After being etched for a defined time, the silica particles were collected by centrifugation and washed several times by using DI water.

\subsection{Drug delivery tests}

p-SiO @void@p-SiO $_{2}$ dry powder (5.0 mg)was placed in a vial containing $10.0 \mathrm{mg}$ R6G in $1.0 \mathrm{~mL}$ water. The mixture was stirred at room temperature for $48 \mathrm{~h}$. The R6G loaded colloids were collected and dried under vacuum overnight, and then dispersed in $100 \mathrm{~mL}$ water and stirred at room temperature. At predetermined time intervals, $0.5 \mathrm{~mL}$ solution was taken from the release medium and centrifuged. The filtrates were then diluted to $1.0 \mathrm{~mL}$ for UV-vis measurement to determine the concentration of R6G released from the colloids.

\subsection{Characterization}

A Tecnai T12 transmission electron microscope was used to characterize the morphology of the product. The annealing process was conducted using an Isotemp programmable muffle furnace (Fisher Scientific). A probe-type Ocean Optics HR2000CGUV-NIR spectrometer was used to measure the transmission spectrum of the solution to monitor the p-SiO $@_{2}$ void@p-SiO ${ }_{2}$ structure evolution. The in vitro release of R6G was also monitored by using the UV -vis spectrometer. Nitrogen adsorption-desorption measurements were carried out on a Micromeritics ASAP 2010 instrument. 


\section{Results and discussion}

The stepwise synthesis of rattle-type p-SiO $@_{2}$ void@ $\mathrm{p}-\mathrm{SiO}_{2}$ colloids is depicted in Fig. 1. Silica seeds with diameter of $\sim 200 \mathrm{~nm}$ were firstly synthesized using a modified Stöber method [18], mixed with PVP solution, and then refluxed at $100{ }^{\circ} \mathrm{C}$ for $3 \mathrm{~h}$ to allow sufficient surface adsorption of PVP molecules. A second layer of silica was then deposited onto the original silica seeds using a sol-gel process similar to the Stöber method [19]. The surface of $\mathrm{SiO}_{2} @ \mathrm{SiO}_{2}$ colloids was also protected by refluxing with PVP solution at $100{ }^{\circ} \mathrm{C}$ for another $3 \mathrm{~h}$. Finally, rattle-type $\mathrm{SiO}_{2} @$ void@SiO ${ }_{2}$ colloids with mesoporous shell and mesoporous movable core could be prepared by etching the PVP protected core-shell colloids using a solution of $\mathrm{NaOH}$ with a relatively high concentration.

The etching process can be well controlled by monitoring the transmittance of the colloidal system. The unprotected silica framework can be etched away gradually by $\mathrm{NaOH}$ solution to form water soluble silicate species [20]. As more silica content dissolves into the alkali solution, the overall transmittance increases accordingly, as shown in Fig. 2(a). The extent of etching of p-SiO $\mathrm{S}_{2}$ void@p-SiO ${ }_{2}$ colloids can therefore be precisely controlled by monitoring the change in the transmittance of the solution. For consistency, we use the transmittance value at a wavelength of $1000 \mathrm{~nm}$ to standardize the monitoring process. Figure 2(b) summarizes the dependence of the transmittance at $1000 \mathrm{~nm}$ on the etching time for a typical $\mathrm{SiO}_{2} @ \mathrm{SiO}_{2}$ sample. In the first $45 \mathrm{~min}$ of reaction, the transmittance changes very slowly because the $\mathrm{NaOH}$ can mainly only attack the surface silica layer which is protected by the PVP molecules. The initial etching does not completely dissolve the surface layer; instead, it creates mesoscale pores on the surface so that the etchant can diffuse inside the particles. Due to the lack of PVP protection, the etching rate of silica underneath the surface layers increases significantly, as suggested by the sharp rise in the curve beyond 45 min (Fig. 2(b)). The entire etching process could be completed within $110 \mathrm{~min}$, which is represented by reaching a transmittance of $\sim 80 \%$ in the spectrum. Comparison with our

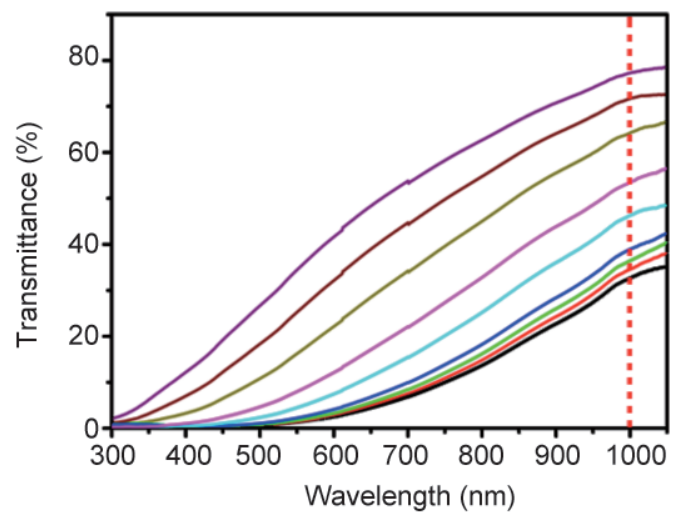

(a)

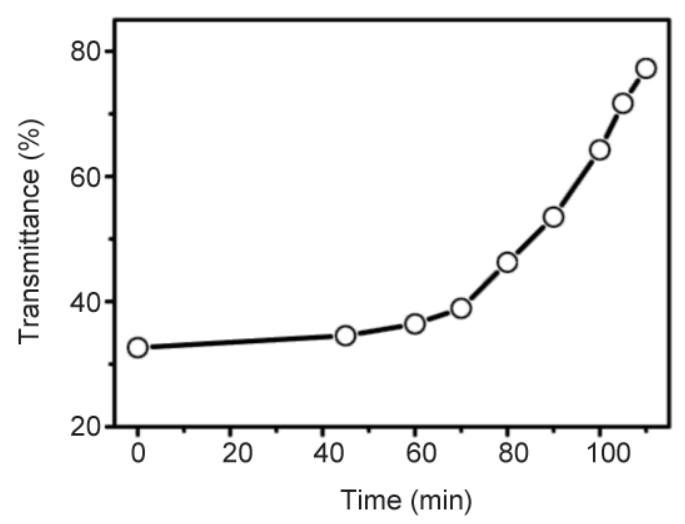

(b)

Figure 2 (a) Optical transmission spectra of a typical sample of $\mathrm{SiO}_{2} @ \mathrm{SiO}_{2}$ core-shell silica colloids during the surface-protected etching process. From the bottom to the top: $t=0,45,60,70$, $80,90,100,105$, and $110 \mathrm{~min}$. The initial core-shell particles were synthesized by coating silica on PVP-treated silica seeds. (b) Dependence of the transmittance at $1000 \mathrm{~nm}$ on the etching time for a typical $\mathrm{SiO}_{2} @ \mathrm{SiO}_{2}$ sample

previous work highlights some useful information about the etching process. In our previous work involving the etching of silica particles synthesized in a single step [7], about $3 \mathrm{~h}$ were required to reach the same transmittance when all the other conditions were kept the same, e.g., reaction temperature, size of silica particles, and PVP pretreatment time. The decreased etching time in this work is probably due to the effectively higher $\mathrm{NaOH} / \mathrm{SiO}_{2}$ ratio because the etching was mainly limited to the outer layer and the inner cores were only minimally etched (if at all).

The degree of etching apparent in the optical transmission measurements is fully consistent with the transmission electron microscopy (TEM) observations. Figure 3 shows TEM images of the original silica seeds and five samples collected at 
different stages during the etching process. The size of the original silica seeds is about $200 \mathrm{~nm}$ (Fig. 3(a)), which increases up to about $400 \mathrm{~nm}$ after coating. When the colloids are etched for only $45 \mathrm{~min}$, there is no obvious contrast between the core and shell (Fig. 3(b)). As the etching time is prolonged to 90 min, the outer shell becomes porous and has lower contrast, while the inner seed appears unchanged (Fig. 3(c)). After about another $10 \mathrm{~min}$, the shell becomes even more porous, with an obvious gap appearing between the shell and the inner core (Fig. 3(d)). After etching for a total of $\sim 110 \mathrm{~min}$, the silica shell becomes thinner and more hollow, such that the interior silica seeds become movable (Fig. 3(e)). When the etching process is allowed to proceed for a longer time, the outer shell is completely etched away and only the inner core remains (Fig. 3(f)).

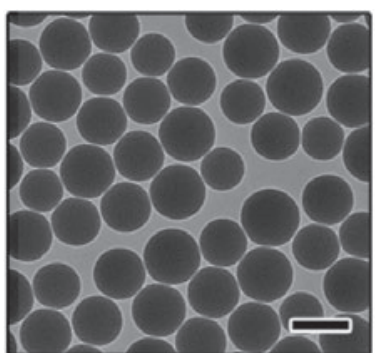

(a)

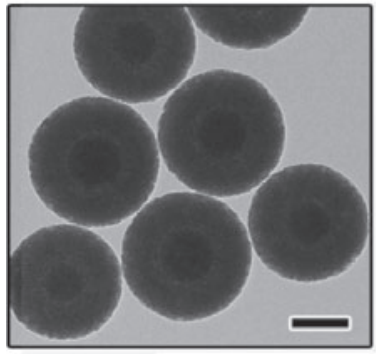

(c)

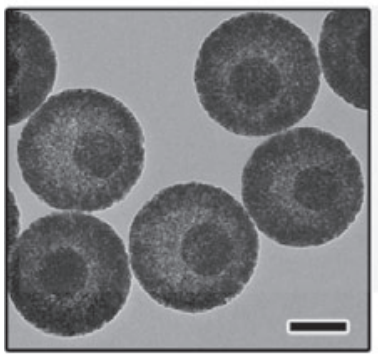

(e)

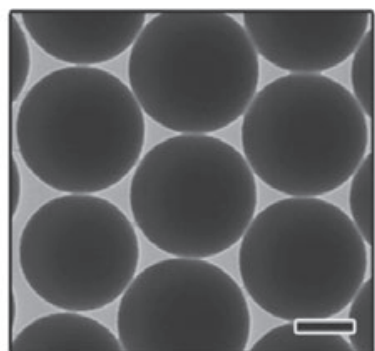

(b)

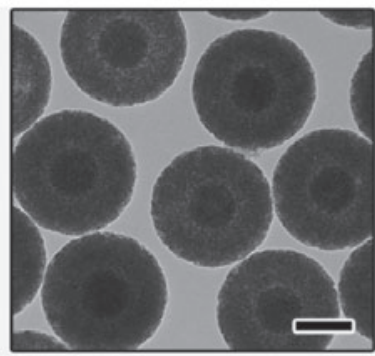

(d)

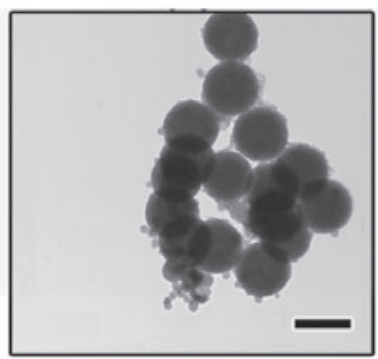

(f)
Figure 3 TEM images of (a) original silica seeds and (b)-(f) $\mathrm{SiO}_{2} @$ $\mathrm{SiO}_{2}$ core-shell colloidal particles collected at different time (from (b) to (f): $45,90,100,110$, and $145 \mathrm{~min}$ ) during the etching process. All scale bars are $200 \mathrm{~nm}$
Although it is difficult to characterize their degree of etching precisely by TEM observation, the inner cores consistently show higher contrast than the outer shells during the hollowing process and their sizes remain essentially unchanged, suggesting their higher resistance against $\mathrm{NaOH}$ etching.

The protection by PVP afforded to both the shell and core can be verified by etching silica particles that had been coated by two additional silica layers. PVP molecules are introduced to the core surfaces before each additional coating. As shown in Figs. $4(\mathrm{a})$ and $4(\mathrm{~b})$, moderate etching with $\mathrm{NaOH}$ firstly produces pores in the outermost shell, and then makes the shell more porous and at the same time

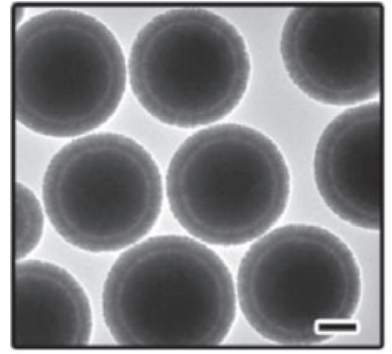

(a)

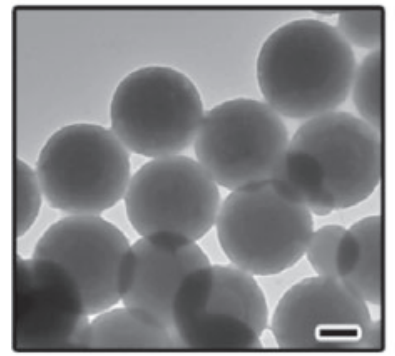

(c)

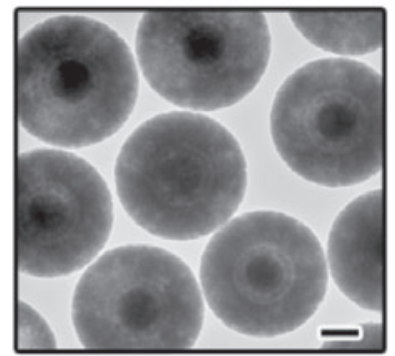

(e)

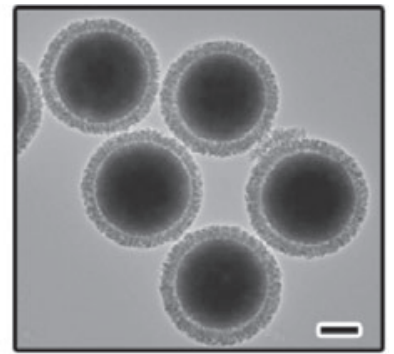

(b)

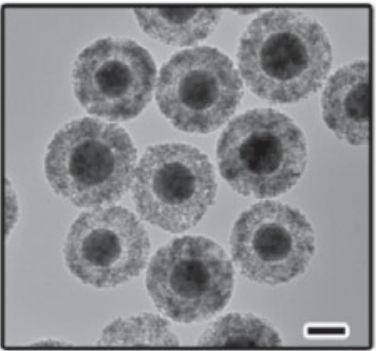

(d)

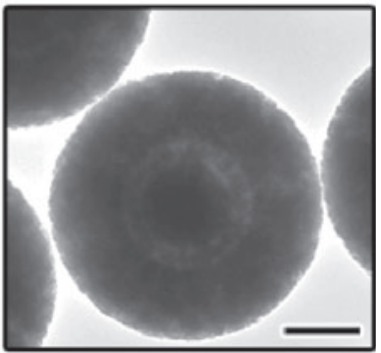

(f)
Figure 4 TEM images of a sample of $\mathrm{SiO}_{2} @ \mathrm{SiO}_{2} @ \mathrm{SiO}_{2}$ colloids collected at different stages of etching: (a) $60 \mathrm{~min}$, (b) $90 \mathrm{~min}$, (c) $120 \mathrm{~min}$, and (d) $160 \mathrm{~min}$. The outermost shell is not thick enough to sustain extensive etching to yield multi-shell rattle structures. (e), (f) TEM images of p-SiO ${ }_{2} @$ void@p-SiO ${ }_{2} @$ void@p- $\mathrm{SiO}_{2}$ multi-layer structures obtained upon etching of another $\mathrm{SiO}_{2} @ \mathrm{SiO}_{2} @ \mathrm{SiO}_{2}$ sample with a thicker outermost shell for $150 \mathrm{~min}$. All scale bars are $200 \mathrm{~nm}$ 
creates a small gap between the shell and core. Further etching of the particles completely removes the outermost shell and exposes the middle silica layer, as shown in Fig. 4(c). Interestingly, continuous etching of the particles converts the middle layer of silica into a hollow shell so that rattle structures are produced again (Fig. 4(d)). This process demonstrates that the PVP molecules sandwiched between silica layers can effectively protect the underlying material from etching. Unfortunately, the outermost layer is not thick enough to sustain extensive etching to yield multi-shell rattle structures. Figures $4(\mathrm{e})$ and 4(f) show our further efforts to create $\mathrm{SiO}_{2} @$ void@ $\mathrm{SiO}_{2} @$ void@SiO${ }_{2}$ multi-layer structures by carefully optimizing the relative thicknesses of the outermost layer and the intermediate layer, suggesting the possibility of fabricating more complicated structures by using the described etching process.

In order to reveal the importance of PVP protection to the creation of the rattle structures, another control experiment was performed by etching $\mathrm{SiO}_{2} @ \mathrm{SiO}_{2}$ colloids that were synthesized without decorating the core surface with PVP. PVP was only used to protect the outer surface. As shown in Fig. 5(a), the overall evolution profile of the transmittance spectrum during the etching process is similar to that in the previous case where the core particle was also protected by PVP. The transmittance change is slow in the initial stage of etching due to the protection of PVP on the outer surface, and then accelerates as the etching process continues. Interestingly, continuous etching for about $3 \mathrm{~h}$ is needed to reach $\sim 80 \%$ transmittance, suggesting that the colloids are etched as a whole and not limited to the shell. This conclusion has also been confirmed by TEM. When the etching process starts, the outer shell becomes porous with lower contrast (Fig. 5(b)). However, no gap between the core and shell can be observed during the entire etching process (Figs. 5(c) and 5(d)). The core appears to be slightly more stable against etching than the surrounding shell as indicated by the apparent contrast in the core and shell, probably because the core has a much longer condensation time before etching. The difference in stability, however, is not significant enough to produce rattle-type structures. With even longer

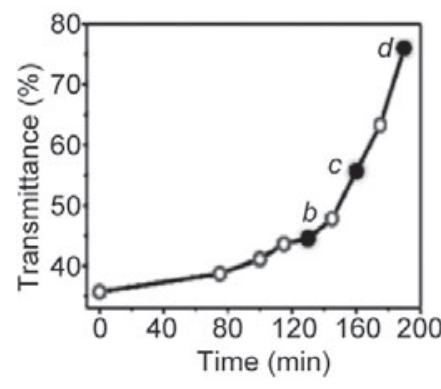

(a)

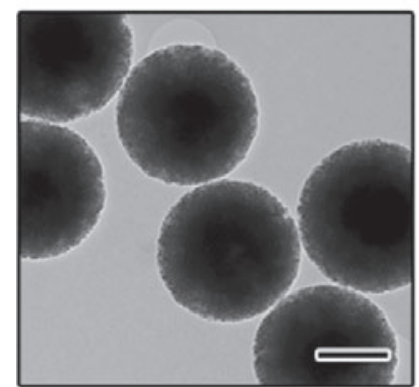

(c)

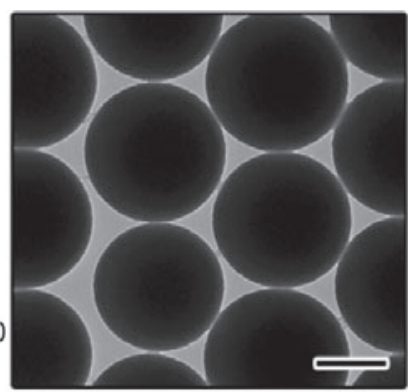

(b)

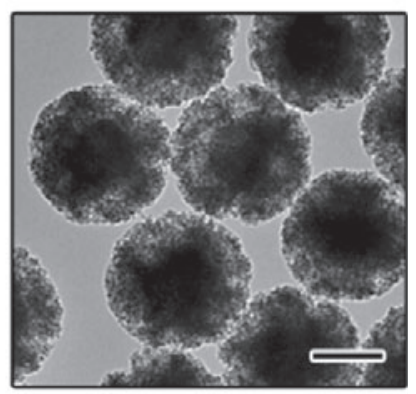

(d)
Figure 5 (a) Optical transmission spectra recorded during the etching of $\mathrm{SiO}_{2} @ \mathrm{SiO}_{2}$ colloids that were synthesized by coating silica on untreated silica seeds. TEM images of $\mathrm{SiO}_{2} @ p-\mathrm{SiO}_{2}$ colloids collected at different transmittance values (b) $45 \%$, (c) $55 \%$, and (d) $79 \%)$ during the etching process. All scale bars are $200 \mathrm{~nm}$

etching time, both the outer layer and the inner seed are dissolved.

We further verified the condensation effect by annealing the silica seeds at $200{ }^{\circ} \mathrm{C}$ for $2 \mathrm{~h}$ prior to the growth of the outer layer. Heating at high temperature favors further polycondensation of the remaining silanol groups in the seeds, and therefore enhances structural stability against chemical etching. As there are fewer silanol groups left on the surface of the seeds, a continuous injection method was used to coat the second silica layer in order to reduce the homogeneous nucleation. A total of $1 \mathrm{~mL}$ TEOS was added over the course of $50 \mathrm{~min}$, increasing the size of particles from $200 \mathrm{~nm}$ to $320 \mathrm{~nm}$. Again, during the etching process, the transmittance increased slowly at the beginning but quickly in the later stages (Fig. 6(a)). As shown in the TEM images in Figs. 6(b)-(d), the inner seeds appear to be more stable because of the heat pretreatment and stay essentially intact as the outer layer is being etched. Although the coreshell structures are distinctive, the gap between the core and the porous shell is not obvious due to the limited shell thickness that is achievable for coating 


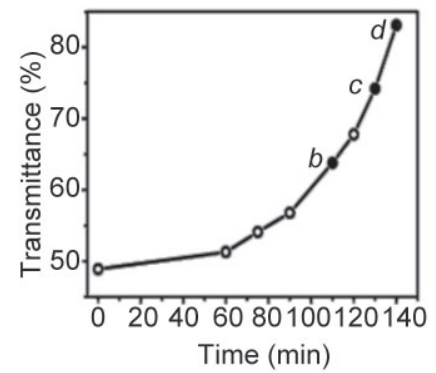

(a)

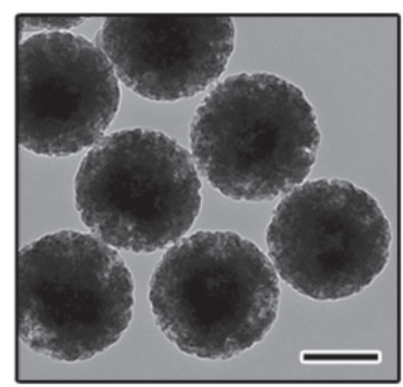

(c)

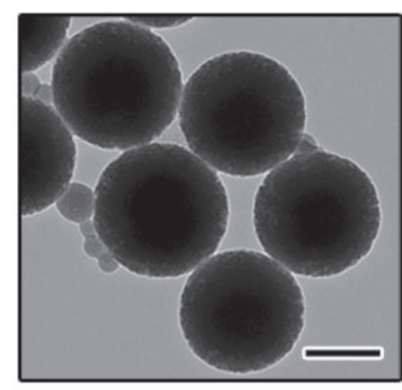

(b)

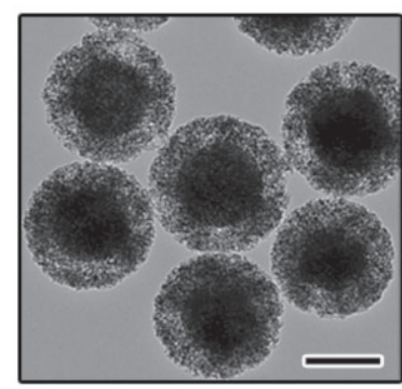

(d)
Figure 6 (a) Optical transmission spectra recorded during the etching of $\mathrm{SiO}_{2} @ \mathrm{SiO}_{2}$ colloids that were synthesized by starting with calcined seeds. TEM images of $\mathrm{SiO}_{2} @ \mathrm{p}-\mathrm{SiO}_{2}$ colloids collected at transmittance values (b) $64 \%$, (c) $74 \%$, and (d) $83 \%$ during the etching process. All scale bars are $200 \mathrm{~nm}$

the heated treated seeds. Using PVP protection of the core surface is therefore still a more convenient and controllable way to produce high quality rattle structures.

To better understand the etching process and the resulting porous structures, nitrogen adsorptiondesorption measurements were carried out on the samples formed by using the surface-protected etching process. Figure 7 shows the representative nitrogen adsorption-desorption isotherms and the corresponding pore size distributions of the samples whose TEM images were displayed in Figs. 3(b) and 3(e). Upon etching for $45 \mathrm{~min}$, the BET (BrunauerEmmett-Teller) surface area and single-point total pore volume were $31.80 \mathrm{~m}^{2} / \mathrm{g}$ and $0.034 \mathrm{~cm}^{3} / \mathrm{g}$, respectively. The surface area is small, implying that the initial etching happens only on the outer surface. The average $\mathrm{BJH}$ (Barrett-Joyner-Halenda) pore diameter calculated from the desorption branch of the isotherm is determined to be $2.4 \mathrm{~nm}$ (Fig. 7(a) inset), while a small proportion of larger pores also exist. At the end of the etching process, both the BET surface area and the average pore size increased, as shown in Fig. 7(b). The BET surface area and singlepoint total pore volume increased to $177.46 \mathrm{~m}^{2} / \mathrm{g}$ and $0.243 \mathrm{~cm}^{3} / \mathrm{g}$, respectively, which are relatively large values. The average $\mathrm{BJH}$ pore diameter was calculated to be $5.6 \mathrm{~nm}$. As shown in the inset in Fig. $7(\mathrm{~b})$, there are two main pore sizes, $6.4 \mathrm{~nm}$ and 2.3 $\mathrm{nm}$. Based on our previous observations, the larger pores are believed to result from the shells that have been etched extensively [20]. The smaller pore size, $2.3 \mathrm{~nm}$ (Fig. 7(b) inset), is close to the value obtained in the initially etched shells, suggesting that the inner core surface has also been partially etched, which is consistent with our TEM observations of a gradual decrease in the contrast of the cores.

Mesoporous silica particles with complex internal structures may find important uses for controlled drug delivery thanks to their excellent thermal

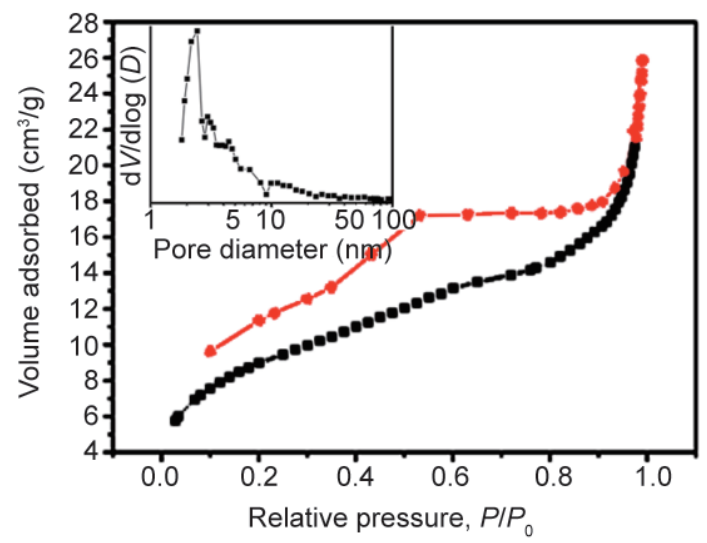

(a)

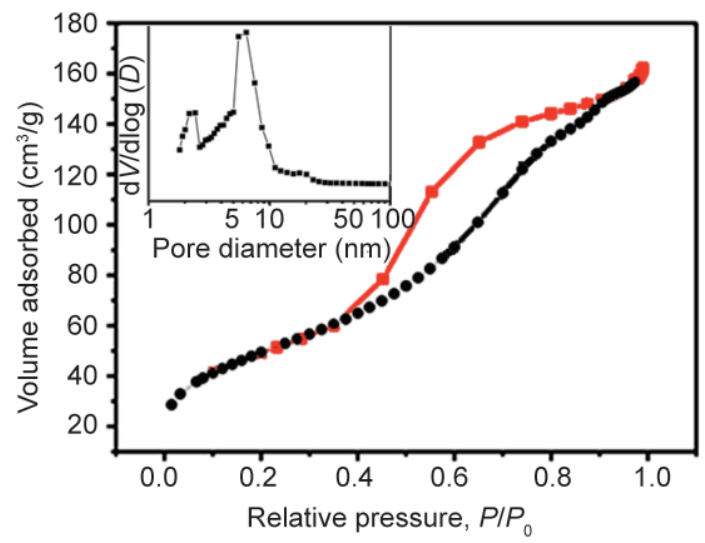

(b)

Figure $7 \quad \mathrm{~N}_{2}$ adsorption/desorption isotherms of the rattle-type structures obtained at different stages of the etching process. Samples (a) and (b) correspond to the particles whose TEM images are displayed in Figs. 3(b) and 3(e), respectively. Insets show the corresponding plots of pore size distribution 
stabilities, chemical durability, large surface areas, and good biocapabilities [21,22]. Here we simply demonstrate the unique drug delivery profile associated with the p-SiO $@$ void@p-SiO ${ }_{2}$ rattle-type colloids by using R6G as a model drug molecule. As shown in Fig. 8, there is an apparent two-stage release pattern of R6G from the rattle structures. The first stage involves the rapid elution of R6G within the first $90 \mathrm{~min}$, followed by a plateau (73.5\%) with only small changes from $90-240 \mathrm{~min}$. We believe that the R6G molecules delivered during this stage are mainly those trapped in the porous silica shell and the inner hollow space. In the second stage, the R6G concentration increases again from 240 to $300 \mathrm{~min}$, and eventually reaches another plateau at $86.3 \%$. It is reasonable to attribute the release in the second stage to the mesoporous silica cores. We believe that the two-step release profile is associated with this particular mesoporous, rattle-type structure because it has been reproducibly observed for several other samples with similar rattle structures. In principle, it is straightforward to modulate the temporal delivery profile and the delivery amount in each stage by changing the relative thickness of core and shells and controlling the degree of hollowness through the surface-protected etching process. A more indepth study along this direction is current underway. Potentially, it is possible to include two or more different drugs in the core and shell regions so that their delivery can be achieved in a programmed

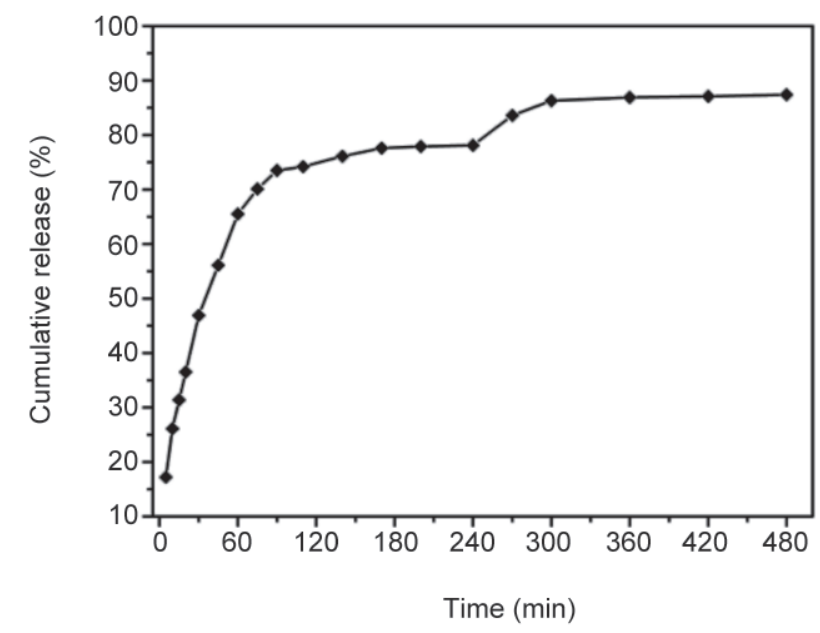

Figure 8 In vitro release profile of R6G from p-SiO ${ }_{2}$ void@p-SiO colloids sequence that can match the physiological requirements of the body, thus minimizing the total dosage of the drugs and preventing the development of biological tolerance and side effects which arise when the drugs are present at their target sites for excessively long time.

\section{Conclusions}

We have demonstrated the unique capability of the "surface-protected etching" process by producing p-SiO $@_{2} @$ void@p-SiO 2 rattle-type colloids with permeable silica shells and movable silica cores. The key in such a process is to introduce a protecting polymer to the particle surface so that only inner regions are removed when the particles are subjected to chemical etching. For core-shell particles, it is possible to create rattle-type structures by protecting the core with a layer of binding polymer and only hollowing the shell layer. While the difference in stability against etching between the inner core and the outer shell can also be achieved by other meansfor example, by thermal annealing of the cores before coating of the shells-the surface protected etching scheme is still the most convenient and reproducible way to obtain rattle structures. As a simple application, the as-synthesized rattle-type colloids have been explored as carriers for controlled drug delivery and shown an interesting and potentially useful two-stage release profile which is believed to be directly related to the particular rattle-type structure. Although this study mainly focuses on the fundamental aspects of the surface-protected etching process, it is expected that the unique rattle-type structures may find wide applications in areas such as catalysis and biomedicine because many active materials can be conveniently incorporated into the porous silica framework to produce novel composite nanostructures with complex morphologies and functions.

\section{Acknowledgements}

Y. Y. thanks the University of California, Riverside for start-up funds and the Chinese-American Faculty Association of Southern California for the Robert T. 
Poe Faculty Development Grant. Acknowledgment is also made to the Donors of the Petroleum Research Fund, administered by the American Chemical Society, for support of this research. We thank Prof. Pingyun Feng and Mr. Rui Liu for assistance with BET measurements.

\section{References}

[1] Sun, X. M.; Liu, J. F.; Li, Y. D. Oxides@C core-shell nanostructures: One-pot synthesis, rational conversion, and Li storage property. Chem. Mat. 2006, 18, 3486-3494.

[2] Zhang, L.; Tu, R.; Dai, H. J. Parallel core-shell metaldielectric-semiconductor germanium nanowires for highcurrent surround-gate field-effect transistors. Nano Lett. 2006, 6, 2785-2789.

[3] Xie, R. G.; Chen, K.; Chen, X. Y.; Peng, X. G. InAs/InP/ ZnSe core/shell/shell quantum dots as near-infrared emitters: Bright, narrow-band, non-cadmium containing, and biocompatible. Nano Res. 2008, 1, 457-464.

[4] Wan, Y.; Min, Y. L.; Yu, S. H. Synthesis of silica/carbonencapsulated core-shell spheres: Templates for other unique core-shell structures and applications in in situ loading of noble-metal nanoparticles. Langmuir 2008 , 24, 5024-5028

[5] Kamata, K.; Lu, Y.; Xia, Y. N. Synthesis and characterization of monodispersed core-shell spherical colloids with movable cores. J. Am. Chem. Soc. 2003, 125, 2384 2385.

[6] Lou, X. W.; Archer, L. A.; Yang, Z. C. Hollow micro-/ nanostructures: Synthesis and applications. Adv. Mater. 2008, 20, 3987-4019.

[7] Zhang, Q.; Zhang, T. R.; Ge, J. P.; Yin, Y. D. Permeable silica shell through surface-protected etching. Nano Lett.

2008, 8, 2867-2871.

[8] Sun, Y. G.; Wiley, B.; Li, Z. Y.; Xia, Y. N. Synthesis and optical properties of nanorattles and multiple-walled nanoshells/nanotubes made of metal alloys. J. Am. Chem. Soc. 2004, 126, 9399-9406.

[9] Yin, Y. D.; Rioux, R. M.; Erdonmez, C. K.; Hughes, S.; Somorjai, G. A.; Alivisatos, A. P. Formation of hollow nanocrystals through the nanoscale Kirkendall effect. Science 2004, 304, 711-714.

[10] Lee, J.; Park, J. C.; Song, H. A nanoreactor framework of a Au@ $\mathrm{SiO}_{2}$ yolk/shell structure for catalytic reduction of p-nitrophenol. Adv. Mater. 2008, 20, 1523-1528.
[11] Yin, Y. D.; Lu, Y.; Sun, Y. G.; Xia, Y. N. Silver nanowires can be directly coated with amorphous silica to generate well-controlled coaxial nanocables of silver/silica. Nano Lett. 2002, 2, 427-430.

[12] Zhang, T. R.; Ge, J. P.; Hu, Y. X.; Zhang, Q.; Aloni, S.; Yin, Y. D. Formation of hollow silica colloids through a spontaneous dissolution-regrowth process. Angew. Chem. Int. Ed. 2008, 47, 5806-5811.

[13] Wang, L.; Zhao, W. J.; Tan, W. H. Bioconjugated silica nanoparticles: Development and applications. Nano Res. 2008, 1, 99-115.

[14] Zhang, T. R.; Zhang, Q.; Ge, J. P.; Goebl, J.; Sun, M. W.; Yan, Y. S.; Liu, Y. S.; Chang, C. L.; Guo, J. H.; Yin, Y. D. A self-templated route to hollow silica microspheres. J. Phys. Chem. C 2009, 113, 3168-3175.

[15] Ikeda, S.; Ikoma, Y.; Kobayashi, H.; Harada, T.; Torimoto, T.; Ohtani, B.; Matsumura, M. Encapsulation of titanium(IV) oxide particles in hollow silica for sizeselective photocatalytic reactions. Chem. Comm. 2007, 3753-3755.

[16] Hu, Y. X.; Ge, J. G.; Sun, Y. G.; Zhang, T. R.; Yin, Y. D. A self-templated approach to $\mathrm{TiO}_{2}$ microcapsules. Nano Lett. 2007, 7, 1832-1836.

[17] Deng, Y. H.; Qi, D. W.; Deng, C. H.; Zhang, X. M.; Zhao, D. $Y$. Superparamagnetic high-magnetization microspheres with an $\mathrm{Fe}_{3} \mathrm{O}_{4} @ \mathrm{SiO}_{2}$ core and perpendicularly aligned mesoporous $\mathrm{SiO}_{2}$ shell for removal of microcystins. $J$. Am. Chem. Soc. 2008, 130, 28-29.

[18] Stöber, W.; Fink, A.; Bohn, E. Controlled growth of monodisperse silica spheres in micron size range. J. Colloid Interface Sci. 1968, 26, 62-69.

[19] Graf, C.; Dembski, S.; Hofmann, A.; Ruhl, E. A general method for the controlled embedding of nanoparticles in silica colloids. Langmuir 2006, 22, 5604-5610.

[20] Ge, J. P.; Zhang, Q.; Zhang, T. R.; Yin, Y. D. Core-satellite nanocomposite catalysts protected by a porous silica shell: Controllable reactivity, high stability, and magnetic recyclability. Angew. Chem. Int. Ed. 2008, 47, 89248928.

[21] Ohta, K. M.; Fuji, M.; Takei, T.; Chikazawa, M. Development of a simple method for the preparation of a silica gel based controlled delivery system with a high drug content. Eur. J. Pharm. Sci. 2005, 26, 87-96.

[22] Liu, R.; Zhao, X.; Wu, T.; Feng, P. Y. Tunable redoxresponsive hybrid nanogated ensembles. J. Am. Chem. Soc. 2008, 130, 14418-14419. 